\title{
Must Structural Realism Cover the Special Sciences?
}

\author{
Holger Lyre
}

\begin{abstract}
Structural Realism (SR) is typically rated as a moderate realist doctrine about the ultimate entities of nature described by fundamental physics. Whether it must be extended to the higher-level special sciences is not so clear. In this short paper I argue that there is no need to 'structuralize' the special sciences. By mounting concrete examples I show that structural descriptions and structural laws certainly play a role in the special sciences, but that they don't play any exclusive role nor that they give us any reason to believe that all that there is on the various levels is structure. I fortify my points by arguing that structures are global entities (in order for SR not to collapse into a bundle ontology) and that the assumption of higher-level structures as genuinely global or holistic entities is even more arcane.
\end{abstract}

Many proponents and opponents of structural realism alike seem to agree on the point that SR, if sound, must provide more than just a metaphysics of the fundamental physical level, but that it should also provide an ontological framework that covers higher levels of complexity and thus pertains to the special sciences as well. As Frigg and Votsis (2011, p. 269) put it:

the question remains whether OSR, and ESR for that matter, can give an adequate account of the ontology and epistemology of other sciences. The bulk of the literature on SR has thus far focussed on modern (and in particular fundamental) physics. This is no accident of history. A structuralist analysis of scientific theories usually departs from those theories' mathematical formalism, and formalisation is the hallmark of modern physics. Therefore SR seems to be at odds with less formal sciences such as biology or the social sciences. This has led some critics to claim that SR is a philosophy with little, if any, relevance outside the province of physics...

\footnotetext{
H. Lyre $(\bowtie)$

Philosophy Department, University of Magdeburg, Magdeburg, Germany

e-mail: lyre@ovgu.de
} 
A few recent authors already took up the challenge and tried to indicate how SR motives can be implemented in the special sciences, as for instance French (2011, 2012) for biology, Kincaid (2008) for social science and Ross (2008) for economy. I consider such attempts as superfluous. In this paper I will argue that there is no need to 'structuralize' the higher-level special sciences. I actually want to show that, quite in contrary, we should not even assume that the special sciences can be provided with the same ontological framework than fundamental physics. In the first section I distinguish between epistemic and ontic SR arguments about the special sciences. I then consider three examples of higher-level structures in the second section. Here my discussion touches on issues of multiple realizability and the possibility of higher-level structural laws. In the third section I argue that OSR is better off to construe structures as global entities to prevent a collapse into a bundle ontology, but that the assumption of higher-level structures as genuinely global or holistic entities is even more arcane. I finish in the fourth section with a short discussion of the possible combinations of SR with scientific (anti-)fundamentalism.

\section{ESR and OSR about the Special Sciences}

Why should one want to extend structural realist motives to the higher-level, more complex sciences? The answer might depend on whether one adopts an epistemic or ontic point of view. Indeed, the vast majority of the more recent authors in the debate about structural realism focuses on OSR. And rightly so, I think. Structural realism is first and foremost an ontological framework that provides us with a tailormade metaphysics for modern physics. I will thus adopt an OSR perspective in this paper as well. To start with, however, I shall briefly consider the title question from an ESR point of view. Epistemic structural realists emphasize that our structuralist conception of the world is due to our peculiar and perhaps limited epistemic access to the world. A higher-level ESRist must therefore find arguments why our access to the world on all levels of complexity is restricted to structures rather than objectlike entities. The point would go through if, indeed, all of our science were basically formulated in terms of structural laws and regularities with non-individual entities and the like. But just the opposite seems to be, at least mostly, the case (pace French, Kincaid and Ross). Quite generally, in our higher-level, special sciences the entities considered on the particular levels (as, for instance, the biological, geological, psychological, sociological, or economic level) are construed as individual entities with intrinsic natures - natures that can be captured by the laws and regularities of the corresponding special science.

Curiously enough, despite prima facie evidence, the above mentioned authors not only want to defend ESR but a stronger and more ambitious OSR about the special sciences. Roughly speaking, special science OSR is the view that all that there is on the various levels of complexity or description on which the special sciences operate is structure. A motive for a proponent of OSR to extend her view to the special sciences is scientific anti-fundamentalism - the belief that there 
is no bottom level. This attitude is sometimes captured by the slogan that it is "structures all the way down". While I think that physics gives us strong reasons to believe in the existence of a bottom level rather than believing in scientific antifundamentalism, I don't delve into arguments for this claim here. What I want to point out is that scientific anti-fundamentalism together with genuine higherlevel OSR commits the proponent of such a view to the existence of 'genuine' structures on all levels. No level is fundamental, no level will serve as the bottom reduction or supervenience base. All levels are, as it were, genuine and consist of bona fide structures. And, of course, it's not only structures all the way down, it's also structures all the way up! Let's see whether the assumptions of such a view are tenable.

\section{Three Examples of Higher-Level Structures}

From now on I focus on OSR, but my point in this section, if successful, can be understood such that even special science ESR is in bad shape (and within the context of our discussion undermining ESR a fortiori undermines OSR as the more ambitious position). My point is the following: while I do believe that structural descriptions and structural laws play an eminent role in the special sciences, I fail to see that they play an exclusive role or that they should give us any reason to believe that all that there is on the various levels is structure. SR proponents don't need to assume the existence of genuine level-bound structures since, in general, higherlevel structures simply supervene on or can otherwise be traced back to lower-level features. I shall present three examples to illustrate this.

Consider, as a first example, the harmonic oscillator. It provides us with a simple and straightforward example of a system that is described by purely structural means. At the same time, and exactly for the same reason, it provides us with a perfect example of multirealizability: harmonic oscillators are instantiated by pendula, springs, electromagnetic circuits, neural circuits etc. All harmonic oscillators are governed by the same structural law: the oscillator equation $\mathrm{d}^{2} / \mathrm{dt}^{2}$ $\mathrm{x}(\mathrm{t})+\mathrm{kx}(\mathrm{t})=0$. It is a structural law in the sense that, as far as the oscillation is concerned, no intrinsic but only relational properties of the target system play a role. That's why harmonic oscillators are multirealizable: they all share the same (sub-) set of relational properties (obeying the same regularity). The various classes of instances of the harmonic oscillator are individuated by the constant $k$ only ( $k$ defines what kind of oscillator we are dealing with: spring, pendulum, neural circuit etc.). The example of the harmonic oscillator expedites an important insight about the nature of multirealizability. As I have argued elsewhere (Lyre 2009), a majority of cases of multirealizability simply rests on the sharing of properties being either intrinsic or even purely relational properties. While cases of shared intrinsic properties are non-thrilling cases of multirealizability (e.g. the property of "being red" is trivially multirealized), harmonic oscillators are of the more interesting type of cases of shared relational properties. 
This directly relates to laws, since laws quantify over shared properties. Take "like charges repel" as a simple example of a law. Like charged particles repel irrespective of their mass, size and spin. So laws quantify over particular properties and disregard others. In this brute sense, laws and in particular higher-level laws are "multirealizable". But these are benign cases of multirealizability of course, since the causally relevant properties are just shared lower-level properties. And this is also true in the case of structural laws such as the oscillator equation (and, as I've argued in my 2009, even in the case of functional laws), where the causally relevant properties are shared lower-level relational properties. But what is most important for the purpose of our discussion is that structural laws, as far as they occur in the special sciences, can be reduced to lower-levels insofar as the purportedly existing higher-level structures simply supervene on lower-level ones (i.e. sets of shared lower-level relational properties).

So let's go over to a second example. I do not want to claim that higher-level structures can in all cases be (Nagel-) reduced to lower-level ones. Surely in many important cases of higher-level structural laws such laws also include epistemically advantageous and tricky approximations and idealizations. Here's a simple toy example for the "addition rules of huge numbers". Consider simple addition cases like $12+36=48$ and $3+3=6=2 \cdot 3$. The general rules can be captured as $a+b=c$, where $c>a, b$ and $a+a=2 a$ with $a, b, c$ being 'small' natural numbers. Now consider the addition of huge numbers like $10^{80}+10^{120} \approx 10^{120}$ and $10^{80}+10^{80}=2 \cdot 10^{80} \approx 10^{80}$. For all practical purposes, the rules for the addition of huge numbers can be generalized as $A+B=B$, where $B>>A$ and $A+A=A$, where $A, B$ are 'huge' numbers (I don't think it's necessary for the purpose of my illustration to define 'small' and 'huge' more rigorously here). What we can see from this simple example is how the higher-level addition structure "emerges", as it were, from the lower-level addition structure of normal and small natural numbers.

My second example can be supplemented by a third one, the example of John Conway's infamous "Game of Life", the probably best-known example of a cellular automaton. 'Life' is fascinating since it opens up a universe of complexity and a plethora of patterns that live on an infinite two-dimensional grid of cells while being based on just three amazingly simple rules. These so-called updating rules for the state of a cell as being either alive or dead simply are: (1) live cells with $n<2$ die (by loneliness), (2) live cells with $\mathrm{n}>3$ die (by overcrowding), and (3) dead cells with $n=3$ come to life ( $n$ is the number of neighbours). The cell patterns that arise during the temporal evolution of a particular game starting from a particular initial state can be seen as higher-level structures that all and only consist of lower-level pixel distributions.

The question arises whether there's ontologically more to such patterns than their undeniable epistemic and pragmatic value from an instrumentalists's and interpretationalist's point of view. Daniel Dennett calls them 'real patterns' (Dennett 1991), but remains vague about the strength of his ontological commitment to them. Ladyman and Ross are more explicit insofar as they subscribe to a scale relativity 
of ontology understood as the thesis that "what (really, mind-independently) exists should be relativized to (real, mind-independent) scales at which nature is measurable" (Ladyman and Ross 2007, p. 200). However, they go on to claim that "because in Life there is an unambiguous fundamental level composed of the aggregation of a finite number of 'little things', and because no higher-level object types cross-classify the dimensions of any models of the game relative to classifications in terms of cells, Life differs greatly from the universe with respect to the kinds of reductionism sustainable in it. Life admits of complete decomposition; the universe does not" (Ladyman and Ross 2007, p. 201, fn. 12). It remains of course their task to prove the latter anti-reductionist claim. ${ }^{1}$

For our purposes, the lesson from the three examples is that structural laws may occur on all levels of complexity and in all domains of science, but that it is very reasonable to assume that they either rest upon shared relational properties (as in the example of the harmonic oscillator) or on approximations or idealizations of lowerlevel properties (as in the second and third example). Under these assumptions, however, higher-level structures aren't bona fide higher-level entities. They are not what a higher-level structuralist must expect them to be.

\section{More Evidence Against Higher-Level OSR: Structures as Global Entities}

OSR must be distinguished from a bundle ontology, where objects are construed as property bundles (non-individual objects may thus be construed as bundles of relational properties). But bundles are still atomistic or pointillistic entities, they are considered to exist at spacetime points. OSR, however, should be conceived as holistic. As I've argued in my 2012, structures aren't mere collections of local relational properties, but global entities reflecting features of the world in toto. Otherwise, OSR collapses to a bundle ontology. At the same time, global structures are in re-structures in the sense that they only exist insofar as they are causally efficacious. For instance, the Lorentz structure of Minkowksi spacetime is causally efficacious insofar as it endows spacetime with a geochronometrical structure of inertial trajectories. Or take the U(1) gauge group. It exists only insofar as it deploys actually occurring causal effects. They can finally be detected in all of our experiments in connection with electromagnetic interactions.

\footnotetext{
${ }^{1}$ A remark about the notorious talk of "cross-classification" (cf. my 2009): the widespread anti-reductionist claim that higher order properties cross-classify lower-level ones, can, as Kim (1998, p.69) has rightly pointed out, only reasonably be maintained if one is willing to give up supervenience. For two taxonomies to cross-classify opens the possibility that the higher-level taxonomic class makes causally efficatious distinctions not made by the lower-level one. But this is a clear failure of supervenience. Cross-classifying taxonomies define conflicting causal profiles.
} 
At least two clarifications are in order here. First, note that the structuralist doesn't want to say that there exist charged particles with interactions but rather that the observations we do in our labs and experiments, and that we superficially attribute to particles, must be traced back to a global U(1) quantum gauge structure of the world that is causally efficacious and, hence, brings about such observable effects. Another way of putting it would be to say that those effects are instantiations or realizations of the global structure. I don't see a particular problem with this phrasing except that many read this as a Platonistic statement: there's an abstract structure on the one hand and it's world-like realization or instantiation on the other. But this, of course, were to confuse in re- with ante rem-structuralism, which is neither intended nor enforced by any of the above. As being in re and concrete, structures should not be confused with abstract Platonistic entities. They are global and concrete rather than local and abstract.

A second point of clarification has to do with "causal efficacy". For a modal structuralist as Esfeld (2012) a structure necessarily brings about its observable effects. For a Humean structuralist as myself the structure just brings about certain effects. They are whatever we observe them to be. No necessity is involved. For both of us, I take it, the structure only exists insofar as its causal efficacy is actualized - meaning that even Esfeld doesn't want to say that there are unactualized dispositional structures. Or does he? Anyway, the Humean point of view give us a straightforward and unambiguous picture: structures just are. We know about their existence because of the observations we do in our labs. These observations can be best explained in terms of a scientific realism of a structuralist kind. No necessity is involved. As being global entities, however, we understand that the observations we do in all of our labs and that are spread over spacetime are orchestrated in such a way that they are the offspring of a global structure and not of local goings-on that must, in a second step, somehow be glued to each other by mysterious modal laws.

Let me come back to my main line of thought. That structures should be considered as global structures provides us with an even stronger argument against special science OSR. It is just highly implausible to assume that higher-level structures reflect genuinely higher-level holistic or global world features. At least, I've never seen arguments in favour of such a view. And notice that a structuralist about higher-level science must show that all levels and, accordingly, all special sciences must be interpreted like this: all levels must then consist of all and only structures and, in order not to collapse structuralism into a bundle view, such structures must be considered as global and holistic structures. Why should anybody subscribe to such an arcane view? Even the proponent of Dennettian real patterns of the Ladyman-Ross-style doesn't commit himself to such patterns as global entities. He rather considers them as patterns that are composed of local objects and their relations (think of the game of life patterns and their composite structure). On higher levels of complexity, our localistic picture of the world (as consisting, for instance of chemical molecules, biological organisms, social groups etc.) is an approximation of a world that supervenes on lower levels, perhaps even on some bottom level. 


\section{Scientific Fundamentalism and OSR}

This finally brings me to the issue of scientific fundamentalism. As I've said in the beginning, a motive for the proponent of OSR to extend his view to the special sciences is scientific anti-fundamentalism. But we've seen arguments against the plausibility of special science OSR. How do things stand if we adopt an OSR perspective together with scientific fundamentalism (in short: F-OSR)?

There are in principle two possibilities here. One might be an F-OSRist and a reductionist and end up with the view that all that there is elementary structure. Basically, that's my favourite view. One might, however, also be an F-OSRist and a non-reductionist. Then there are again two possibilities. One might think that only the bottom level consists of structures while all the higher levels consist of object-like entities (or a mixture of object-like entities and structures). In a sense that sounds like a strange and hybrid position. Perhaps a proponent of such a view believes that there exist higher-level object-like entities with intrinsic natures and that it is generally impossible to reduce intrinsic to relational properties. But that is obviously wrong, intrinsic properties may very well supervene on relational properties. Here's a simple example: the property of "being a graph" can be considered an intrinsic property of the whole graph, which, itself, is a purely structural entity. ${ }^{2}$ In any case, such a position is not in my present scope since we are interested in the question whether there's any need for higher-level structures.

So, as a second possibility, one might think that both the bottom and all of the higher levels consist of structures. But in that case my preceding objections already apply. And in a sense they apply even stronger. While the anti-fundamentalist will typically also be an anti-reductionist, the F-OSRist has now, in the light of the preceding objections, good reasons to give up anti-reductionism. So, again, we are better off with reductionist F-OSR.

\footnotetext{
${ }^{2}$ Anyway, OSR is more than the idea that there are "just relations". As I've argued elsewhere (Lyre 2010, 2012), OSR must be supplemented with a weak and special type of intrinsicality. I've dubbed this 'Extended OSR' (ExtOSR) - the view that relational and structurally derived intrinsic properties exist. Simple OSR, by contrast, assumes only relational but no intrinsic properties (however, both ExtOSR and SimpOSR are versions of non-eliminative OSR). Reasons to prefer ExtOSR over SimpOSR are symmetry invariants and zero-value properties (cf. my 2012). Pick up the first reason: The symmetry invariants under a given symmetry over a domain $\mathrm{D}$ provide properties that are shared by all members of D. They are 'intrinsic' in the sense that they belong to all members of D irrespective of the existence of other objects. Since they are shared by all members of D, they serve to individuate domains, not individuals. Such structure invariants provide structurally derived intrinsic properties. SimpOSR denies intrinsicality, but symmetry groups come equipped with their invariants. So SimpOSR doesn't have the resources to embrace the symmetry structures of modern physics represented by the fundamental symmetry groups. Moreover, almost all fundamental symmetries are quantum gauge symmetries. Here, the argument about symmetry invariants becomes even more pressing since gauge symmetry transformations possess no real instantiations. Only the gauge invariants do. Hence, ExtOSR must be favoured.
} 


\section{Conclusion}

I've granted the occurrence of higher-level structural laws, but at the same time I've argued that such higher-level structural laws may typically be understood as supervening on lower-level properties or structures. Nothing commits us to the existence of genuine, non-reducible higher-level structures. I've fortified my points by arguing that structures are global entities and that the assumption of higher-level structures as genuinely global or holistic entities is even more arcane. It is only on the bottom level, where the global and holistic nature of the fundamental structures becomes apparent. It is, accordingly, only reductionist F-OSR that provides us with a genuine structuralism that doesn't collapse to a bundle view. There's all in all no need to structuralize the special sciences.

\section{References}

Dennett, D. (1991). Real patterns. Journal of Philosophy, 88(1), 27-51.

Esfeld, M. (2012). Causal realism. In D. Dieks et al. (Eds.), Probabilities, laws, and structures (pp. 157-168). Dordrecht: Springer.

French, S. (2011). Shifting to structures in biology and beyond: A prophylactic for promiscuous realism. Studies in History and Philosophy of Biological and Biomedical Sciences, 42, 164-173.

French, S. (2012). The resilience of laws and the ephemerality of objects: Can a form of structuralism be extended to Biology? In D. Dieks et al. (Eds.), Probabilities, laws, and structures (pp. 187-199). Dordrecht: Springer.

Frigg, R., \& Votsis, I. (2011). Everything you always wanted to know about structural realism but were afraid to ask. European Journal for Philosophy of Science, 1(2), 227-276.

Kim, J. (1998). Mind in a physical world. Cambridge, MA: MIT Press.

Kincaid, H. (2008). Structural realism and the social sciences. Philosophy of Science, 75, 720-731.

Ladyman, J., \& Ross, D. (2007). Everything must go: Metaphysics naturalized. Oxford: Oxford University Press.

Lyre, H. (2009). The "multirealization” of multiple realizability. In A. Hieke \& H. Leitgeb (Eds.), Reduction - Abstraction - Analysis (pp. 79-94). Frankfurt: Ontos.

Lyre, H. (2010). Humean perspectives on structural realism. In F. Stadler (Ed.), The present situation in the philosophy of science (pp. 381-397). Dordrecht: Springer.

Lyre, H. (2012). Structural invariants, structural kinds, structural laws. In D. Dieks et al. (Eds.), Probabilities, laws, and structures (pp. 179-191). Dordrecht: Springer.

Ross, D. (2008). Ontic structural realism and economics. Philosophy of Science, 75, 731-741. 